\title{
The behavior of solutions of multidimensional aggregation equations with mildly singular interaction kernels
}

\author{
Andrea L. Bertozzi* ${ }^{\dagger} \&$ Thomas Laurent* $* \ddagger$
}

May 29, 2009

\begin{abstract}
We consider the multidimensional aggregation equation $\partial_{t} \rho-\operatorname{div}(\rho \nabla K * \rho)=0$ in which the radially symmetric attractive interaction kernel has a mild singularity at the origin (Lipschitz or better). We review recent results on this problem concerning well-posedness of nonnegative solutions and finite time blowup in multiple space dimensions depending on the behavior of the kernel at the origin. We consider the problem with bounded initial data, data in $L^{p} \cap L^{1}$, and measure-valued solutions.
\end{abstract}

*Department of Mathematics, University of California - Los Angeles, Los Angeles, California 90095, USA

${ }^{\dagger}$ E-mail: bertozzi@math.ucla.edu, Internet: http://www.math.ucla.edu/ bertozzi

${ }^{\ddagger}$ E-mail: laurent@math.ucla.edu, Internet: http://www.math.ucla.edu/ laurent 


\section{Introduction}

The study of active scalar problems has roots in both classical problems in fluid dynamics, such as $2 \mathrm{D}$ vortex dynamics [40,52] and quasi-geostrophic equations [25]. At the same time there is a body of work in which the scalar generates a gradient flow which can lead to aggregative or dispersive behavior $[47,48]$. The general problem can be written as

$$
\frac{\partial \rho}{\partial t}-\operatorname{div}(\rho \vec{v})=0, \quad \vec{v}=\vec{K}_{s} * \rho+\nabla K * \rho, \quad \rho_{t=0}=\rho_{0}(x) \geq 0, \quad x \in R^{n}
$$

where $\rho$ is convected by a vector field obtained from itself via a convolution operator. The general velocity field has two unique components - a divergence free part, $K_{s} * u$, and a gradient part, $\nabla K * u$, which we focus on here. Examples of the former case include the vorticity-stream form of the Euler equations in 2D, for which $K_{s}$ is the well-known Biot-Savart kernel $\frac{1}{2 \pi} \frac{x^{\perp}}{|x|^{2}}$, and quasi-geostrophic models for which $K_{s}=\nabla^{\perp}(-\Delta)^{-\alpha}$ involving the fractional Laplacian. The model with both incompressible and gradient flow parts was proposed in $2 \mathrm{D}$ for vortex motion in superconductors [27] and for flocking problems [47] as a generalization of $1 \mathrm{D}$ nonlocal swarming models [42]. The purely gradient flow case has been studied for self-interacting individuals via pairwise potentials arising in the modelling of animal collective behavior: flocks, schools or swarms formed by insects, fishes and birds. The simplest models based on ODEs systems [15, 24, 29, $43,44]$ led to continuum descriptions $[19,18,14,37,42,47,48]$ for the evolution of densities of individuals. It is this class of models that we focus on here, although we will draw parallels to well-known problems and results from the incompressible flow literature.

For simplicity, we refer to the gradient flow problem as an 'aggregation equation' taking the form

$$
\frac{\partial \rho}{\partial t}-\operatorname{div}(\rho \nabla K * \rho)=0, \quad \rho_{t=0}=\rho_{0} \geq 0 .
$$

This model shares some features with the classical Patlak-Keller-Segel model for chemotaxis $[33,46]$ without diffusion, see $[16,12,13,26]$ for the state of the art in this problem. Here, the main similarity is the possible formation of a finite time point concentration and the main difference the strong singularity of the potential in the Patlak-Keller-Segel system. Following estimates from the quasi-geostrophique literature, equation (1.2) with additional fractional diffusion also has some prior and recent study in the literature, namely $[11,35,36]$. In the case of fractional diffusion and Lipschitz kernels, there is a critical diffusion exponent for which the solution no longer blows up in finite time.

In this paper, we focus on the case involving only attractive forces $[7,9,34,47]$ and no diffusion. Individuals attract each other under the action of a radially symmetric Lipschitz interaction potential $K(x)=k(|x|)$ with $k(r)$ increasing in $r$, smooth away from zero and bounded

below. Since potentials are defined up to constant, we assume without loss of generality that $k(0)=0$. Some examples appearing in applications are $K_{1}(x)=1-e^{-|x|}, K_{2}(x)=1-e^{-|x|^{2}}$, and $K_{\alpha}(x) \simeq|x|^{\alpha}$ locally near 0 with $\alpha \geq 1$.

This class of equations belongs to the same family of nonlinear friction equations that appear in the modelling of granular media [6, 21, 22, 38, 49]. In those references, several results regarding the long time asymptotics and rates of equilibration were obtained in cases in which the potential $K(x)$ is smooth and convex. In our typical cases, convexity fails. In fact, the equation (1.2) can be formally considered as a gradient flow of the energy functional:

$$
E(\rho)=\frac{1}{2} \int_{\mathbb{R}^{N}} \int_{\mathbb{R}^{N}} K(x-y) \rho(x) \rho(y) d x d y
$$


with respect to the Euclidean Wasserstein distance as introduced in [45] and generalized to a large family of PDEs in $[3,22]$. Its connection to optimal transport theory comes from the convexity properties of the energy functional with respect to geodesic convexity in this distance, called displacement convexity [41]. A nice introduction to this different point of view can be seen in [50].

In this paper we review some recent results from the literature - in particular the role of the Osgood property for the potential,

$$
\int_{0}^{1} \frac{d r}{k^{\prime}(r)}=\infty
$$

which guarantees global existence of bounded solutions and solutions in some $L^{p}$ spaces. Simultaneously, when the Osgood condition in violated,

$$
\int_{0}^{1} \frac{d r}{k^{\prime}(r)}<\infty
$$

then solutions blow up in finite time. Note that the number $\int_{0}^{1} \frac{d r}{k^{\prime}(r)}$ has a natural interpretation: it can be thought as the time it takes for a particle obeying the ODE $\dot{x}=-\nabla K(x)$ to reach the origin if it starts at a distance 1 from the origin. This number quantifies the attractive strength of the potential: the smaller it is, the more attractive the potential is. We discuss this in more detail in later sections.

This review article is organized as follows: Section 2 reviews the discrete particle problem which motivates results in sections 3,4 , and 5 on the continuum problem and blowup. Section 3 reviews recent results connecting the Osgood condition to global and local existence of solutions of the continuum equations, from bounded initial data. Section 4 discusses the shape of the blowup in the special case of kernel $K(x)=|x|$, which arises in a number of models from biology and materials science. Section 5 reviews the recent well-posedness theory for general measure solutions. Section 6 discusses the special case of solutions with initial data in $L^{p}$, and critical $p$ for local well-posedness.

\section{The discrete particle problem}

When the solution is represented by $L$ particles $\left\{x_{1}, \ldots, x_{L}\right\}$ of respective mass $\left\{m_{1}, \ldots, m_{L}\right\}$ the evolution equation reduces (at least formally) to a coupled set of ODEs for the particle paths:

$$
\frac{d x_{i}}{d t}=-\sum_{j \neq i} m_{j} \nabla K\left(x_{i}-x_{j}\right)=-\sum_{j \neq i} m_{j} \frac{x_{i}-x_{j}}{\left|x_{i}-x_{j}\right|} k^{\prime}\left(\left|x_{i}-x_{j}\right|\right), \quad i=1, \ldots, L,
$$

with $x_{i}(t) \in \mathbb{R}^{N}$ for all $t \geq 0$. Note that these equations preserve the total mass $M:=\sum_{j} m_{j}$ of the system and the center of mass $c_{M}:=\sum_{j} x_{j} m_{j} /\left(\sum_{j} m_{j}\right)$. The latter is true because of the symmetry of $K$. Assume that the $L$-particles with total mass $M$ and zero center of mass are initially inside the ball of radius $R_{0}$. Denote by $R(t)$ the distance between the center of mass and the particle situated the furthest apart from the center of mass, i.e., $R(t)=\left|x_{i}(t)-c_{M}\right|=\left|x_{i}(t)\right|$ with $i$ being its label. Thus, due to (2.6), we have

$$
\frac{d}{d t} R(t)^{2}=\frac{d}{d t}\left|x_{i}\right|^{2}=-2 \sum_{j \neq i} m_{j} \frac{\left(x_{i}-x_{j}\right) \cdot x_{i}}{\left|x_{i}-x_{j}\right|} k^{\prime}\left(\left|x_{i}-x_{j}\right|\right) .
$$


Since the $i$ th particle is the one furthest away from the center of mass, we have that $\left(x_{i}-x_{j}\right) \cdot x_{i} \geq 0$ and that $\left|x_{i}-x_{j}\right| \leq 2 R(t)$ for $j \neq i$. Assume that

$$
\frac{k^{\prime}(r)}{r} \text { is decreasing for } r>0 .
$$

Putting together the previous information, we deduce

$$
\frac{d}{d t} R(t)^{2} \leq-\frac{k^{\prime}(2 R(t))}{R(t)} \sum_{j \neq i} m_{j}\left(x_{i}-x_{j}\right) \cdot x_{i} .
$$

Due to conservation of mass and center of mass, we get

$$
\sum_{j \neq i}\left(x_{i}-x_{j}\right) \cdot x_{i} m_{j}=\sum\left(x_{i}-x_{j}\right) \cdot x_{i} m_{j}=M\left|x_{i}\right|^{2}=M R(t)^{2}
$$

and thus,

$$
\frac{d}{d t} R(t) \leq-\frac{M}{2} k^{\prime}(2 R(t))
$$

If the potential $K(x)=k(|x|)$ satisfies the non-Osgood condition (1.5), then the ODE $d R / d t=$ $-M k^{\prime}(2 R) / 2$ with initial data $R=R_{0}$ touches down to zero in finite time, and therefore the particles aggregate in a single particle with the total mass $M$ located at the center of mass before the touch-down time of the ODE (2.8). This time is uniform for particles inside a fixed ball of radius $R_{0}$ initially with total mass $M$. This argument is inspired by and extends previous work in the control theory literature on cooperative motion with first order control laws involving pairwise interaction potentials (see [24] for the case of attractive-repulsive potentials and [28] for quadratic potentials). The argument is proved rigorously in the following theorem:

Theorem 2.1 (Collapse of the ODEs [8]) Consider the ODE system (2.6) satisfying $k^{\prime}(r) / r$ monotone decreasing, with $k^{\prime \prime}(r)$ defined and nonnegative on $(0, \infty)$. If $K$ satisfies the Osgood condition (1.4) then there exists a unique global-in-time forward solution with no collisions, in which the particles converge to their center of mass in infinite time. If $K$ satisfies the non-Osgood condition (1.5) then there exists a unique global-in-time forward solution with collisions, in which the particles all merge at their center of mass in finite time. In the latter case, for a given potential, an upper bound on the merger time is a function of the radius of support of the initial data and the total mass only.

Remark 2.2 (Non-uniqueness) We note that in the non-Osgood case, uniqueness of the ODE does not hold going backward in time because merger of particles destroys information. This is a classical result of uniqueness of solutions of ODEs [4]. This is, in some sense, analogous to shocks in conservation laws, where information goes into the shock and is lost afterwards. In the case of Osgood vector fields, the solution is unique going forward and backward in time. For linear equations, existence of signed measure-valued solutions is recently discussed in the literature [2]. Here we consider a nonlinear problem where the solution itself determines the transporting vector field (the hallmark of an active scalar problem). In Section 5 we discuss uniqueness of measure-valued solutions of the nonlinear problem using optimal transport theory and the gradient structure of the problem.

In the next subsection we show how this collapsing support argument can be used to prove finite time blowup of the continuum problem in the case of non-Osgood potentials. We consider bounded initial data, therefore the characteristic paths are smoother than the point particle case considered in this subsection. However we can still implement the estimate on the size of the support of the solution, proving finite time blowup of the continuum problem. 


\section{The continuum problem with bounded data}

\subsection{Blowup for non-Osgood potentials}

Let us first review the well-posedness of the continuum problem with bounded data. We build primarily on the work of $[7,9,34]$. These papers establish the existence and uniqueness theory for (1.2) in dimensions two and higher, in the case of an acceptable potential satisfying the following criteria:

Definition 3.1 ([34]) The potential $K$ on $\mathbb{R}^{N}, N \geq 2$ is acceptable if $\nabla K \in L^{2}\left(\mathbb{R}^{N}\right)$ and $\Delta K \in$ $L^{p}\left(\mathbb{R}^{N}\right)$ for some $p \in\left[p^{*}, 2\right]$, where $\frac{1}{p^{*}}=\frac{1}{2}+\frac{1}{N}$. In the case of compactly supported initial data, we can take $\nabla K \in L_{\mathrm{loc}}^{2}\left(\mathbb{R}^{N}\right)$ and $\Delta K \in L_{\mathrm{loc}}^{p}\left(\mathbb{R}^{N}\right)$.

We note that the typical kernels considered in this paper satisfy the acceptability condition. In particular, $K$ Lipschitz satisfies $\nabla K$ bounded a. e. and thus is in $L_{\text {loc }}^{2}\left(\mathbb{R}^{N}\right)$. Moreover, the most singular case at the origin is $\Delta K \sim \frac{1}{|x|}$ which satisfies the $L^{p}$ condition above in dimensions two and higher. The case of one space dimension has special issues and we discuss that at the end of this section.

The continuum model assumes a nonnegative density $\rho(t, x)$ at position $x \in \mathbb{R}^{N}$ and time $t>0$ satisfying

$$
\left\{\begin{aligned}
\frac{\partial \rho}{\partial t}(t, x)+\operatorname{div}[\rho(t, x) v(t, x)] & =0 & & t>0, x \in \mathbb{R}^{N}, \\
\text { with velocity field } v(t, x) & :=-\nabla K * \rho(t, x) & & t>0, x \in \mathbb{R}^{N}, \\
u(0, x) & =u_{0}(x) \geq 0 & & x \in \mathbb{R}^{N} .
\end{aligned}\right.
$$

where $v$ is the velocity field under which individuals in the swarm are moving obtained through the "averaging" of the pairwise potential by the distribution of mass.

It is clear that solutions of (3.9) formally preserves the total mass of the system

$$
\int_{\mathbb{R}^{N}} \rho(t, x) d x=\int_{\mathbb{R}^{N}} \rho_{0}(y) d y:=M
$$

and the center of mass

$$
\int_{\mathbb{R}^{N}} x \rho(t, x) d x=\int_{\mathbb{R}^{N}} x \rho_{0}(y) d y:=M c_{M}
$$

where for the last one, we use that $\nabla K$ is anti-symmetric, $\nabla K(-x)=-\nabla K(x)$. We now review the well-posedness theory for $H^{s}$-solutions.

Theorem 3.2 (Existence theory for $H^{s}$ data $[\mathbf{9}, 34]$ ) Given initial data $\rho_{0} \in H^{s}\left(\mathbb{R}^{N}\right), N \geq$ 2 , for positive integer $s \geq 2$, there exists a unique weak solution $\rho(x, t)$ of $(3.9)$ and a maximal interval of existence $\left[0, T^{*}\right)$ such that either $T^{*}=\infty$ or $\lim _{t \rightarrow T^{*}} \sup _{0 \leq \tau \leq t}\|\rho(\cdot, \tau)\|_{L^{q}}=\infty$. The result holds for all $q \geq 2$ for $N>2$ and $q>2$ for $N=2$.

It is shown in [9] that as long as the $L^{q}$-norm of the solution is bounded, then the $H^{s}$-norm of the solution must also remain bounded [9, Proposition 2]. In other words, the $L^{q}$-norm controls the $H^{s}$-norm. This is why in the above theorem the eventual blow-up first occurs in $L^{q}$.

When the kernel $K$ is $C^{2}$, one can derive an a priori bound for $\rho$ in $L^{\infty}$ (see [47,34]) thereby guaranteeing global existence of an $H^{s}$ solution. Moreover, when the kernel has a Lipschitz 
point at the origin, for example the Morse potential $K(x)=1-e^{-|x|}$, one can have finite time blowup. The proof in [9] uses the energy (1.3) and provides an a priori lower bound for $E$ while simultaneously proving an a priori upper bound for the rate of decrease for the energy $E$ when the data is radially symmetric and smooth. More recently these results have been extended in [7] to the case of solutions with (weaker) initial data in $L^{1} \cap L^{\infty}$. With mild decay conditions at infinity and the same conditions on the kernel $K$ as above, we have local in time well-posedness of the problem and continuation of solutions. For simplicity we state the result for data with compact support.

Theorem 3.3 (Existence theory for $L^{1} \cap L^{\infty}$ data [7]) Given compactly supported initial data $\rho_{0} \in L^{1}\left(\mathbb{R}^{N}\right) \cap L^{\infty}\left(\mathbb{R}^{N}\right), N \geq 2$, there exists a unique weak solution $\rho(x, t)$ of $(3.9)$ and a maximal interval of existence $\left[0, T^{*}\right)$ such that either $T^{*}=\infty$ or $\lim _{t \rightarrow T^{*}} \sup _{0 \leq \tau \leq t}\|\rho(\cdot, \tau)\|_{L^{q}}=\infty$. The result holds for all $q \geq 2$ for $N>2$ and $q>2$ for $N=2$.

Existence of solutions for $L^{1} \cap L^{\infty}$ data is proved by constructing first the characteristics for the weak problem. This approach requires unique solutions to the characteristic equation, which requires a certain degree of regularity of the velocity field $v$. Provided $\rho$ is bounded, it is shown in [7] that $v$ is Lipschitz continuous and moreover $\operatorname{div}(v)$ is $\log$-Lipschitz continuous (Lipschitz continuous) in dimension two (three and higher).

Since the mass of the solution is conserved on its interval of existence, another way to prove finite time blowup is to derive an estimate for the size of the support of the solution. If an upper bound for the size of the support shrinks to zero in finite time, this also guarantees that the time interval of existence of the $L^{1} \cap L^{\infty}$ solution is less than infinity. We will now show how to extend the analysis from the ODE case (section 2 of this paper) to the continuum problem.

Proposition 3.4 (Frozen-in-time velocity estimate [8]) Assume $k^{\prime}(r) / r$ is a monotone decreasing function of $r$. Consider a nonnegative function $\rho: \mathbb{R}^{N} \rightarrow \mathbb{R}$ with total mass $M$, first moment zero and compact support. Consider any $B_{R}(0)$ containing the support of $\rho$. Then, for any $x \in \partial B_{R}(0)$ we have

$$
v(x) \cdot x \leq-\frac{k^{\prime}(2 R) R}{2} M \leq 0 .
$$

where $v=-\nabla K * \rho$.

The above proposition is now used to prove the following theorem. This is a generalization of [9, Theorem 6] and [7, Theorem 6.2] to the the case of less singular kernels satisfying (1.4) and the monotonicity conditions in Proposition 3.4. Also, significantly, the radial symmetry of the initial data, required in the proofs from $[9,7]$ is no longer necessary.

Theorem 3.5 (Finite time blowup for compactly supported solution in $L^{\infty}[8]$ ) Let $\rho$ be a weak solution of (3.9) with nonnegative compactly supported initial data in $L^{\infty}\left(\mathbb{R}^{N}\right)$. Let $K$ satisfy the conditions (1.5) and $k^{\prime}(r) / r$ monotone decreasing, $k^{\prime}(r)>0$. Then there exists a maximal time $T^{*}<\infty$ and a unique weak solution $\rho$ to the problem (3.9) on the interval $\left[0, T^{*}\right)$. Moreover

$$
\lim _{t \rightarrow T^{*}} \sup _{0 \leq \tau \leq t}\|\rho(\cdot, \tau)\|_{L^{q}}=\infty \quad \text { for } q \in[2, \infty] \text { if } N>2 \text { and } q \in(2, \infty] \text { if } N=2 \text {. }
$$

Proof. Given the existing continuation theorem, it suffices to prove that the solution ceases to exist in finite time. To do that, we prove a comparison principle for the support of the solution: 
Proposition 3.6 (Comparison principle [8]) Let $\rho(x, t)$ be the weak solution in Theorem 3.5. Let $B_{R_{0}}\left(c_{M}\right)$ contain the support of the solution at time zero. Let $\tilde{R}(t)$ be the unique solution of the ordinary differential equation $d R / d t=-M k^{\prime}(2 R) / 2$. On any time interval of existence of the $L^{1} \cap L^{\infty}\left(\mathbb{R}^{N}\right)$ solution $\rho(x, t)$, the support of $\rho$ must lie inside $B_{\tilde{R}(t)}\left(c_{M}\right)$.

We briefly discuss the aggregation equation in one space dimension. This case is somewhat special. First of all, $k^{\prime \prime}$ plays an important role in the blowup dynamics because the amplification factor for $\rho$ along characteristics is $k^{\prime \prime} * \rho$. In the case of a kernel with a Lipschitz point at the origin, one has finite time blowup because $k^{\prime \prime}$ is a delta function to leading order, and thus the blowup is driven by a quadratic function of $\rho$. This argument was presented in [30] and made rigorous in [14]. For power-law potentials smoother than the Lipschitz case, one can read the paper by $\mathrm{Li}$ and Toscani [38]. In both $[14,38]$ the transformation $w=\int_{-\infty}^{x} \rho$ is used resulting in a nonlocal scalar conservation law for $w$ :

$$
w_{t}-k^{\prime \prime} * w w_{x}=0 \text {. }
$$

We see now that when $k^{\prime \prime}$ is a delta, the problem reduces to Burgers equation and the blowup is simply shock formation in $w$. For more regular kernels than Lipschitz, one needs an existence theory. The work of [34] proves local existence in one dimension for sufficiently smooth initial data and kernels satisfying $k^{\prime \prime}=C \delta+P$ where $P$ is $L^{1}$. To the best of our knowledge, the full existence and continuation theory for general $K$ and bounded initial data, in 1D, has not been derived in the most general setting - however the a priori bounds presented in this section still hold and apply to this problem. For completeness, we remind the reader that in dimensions two and higher, if the kernel $K$ has a Lipschitz point at the origin, then $\Delta K *$ as a convolution operator provides additional smoothness (typically a gain of $N-1$ derivatives in dimension $N$ ), that is lacking in one dimension.

\subsection{Global existence of solutions for Osgood potentials}

In this section we review recent results for global existence of solutions in the case of Osgood potentials satisfying monotonicity conditions. To do this, we obtain refined estimates on the $L^{\infty}$-norm of $\operatorname{div}(v)$. Note first that the Osgood condition is more general than $K \in C^{2}$. For example, $K(x)=|x|^{2}|\ln | x||$ satisfies this condition. Moreover, one does not, in general, have boundedness of $\operatorname{div}(v)$ whenever the density is given by a general nonnegative measure $\mu$, so that one has to rely on the nonlinearity in the evolution equation to provide an a priori bound for $\|\rho\|_{L^{\infty}}$. For example, if $v$ is log-Lipschitz then the modulus of continuity $\rho$ only guarantees particle paths that are Hölder continuous, which is insufficient to guarantee that they would map $L^{\infty}$ densities to $L^{\infty}$. Instead, we have to examine the evolution equation and use the fact that a smoother density yields a more regular velocity field.

We begin by reviewing the $C^{2}$ case, which has already been studied in the literature. Along characteristics, we have $\partial_{t} \rho+v \cdot \nabla \rho=-\rho \operatorname{div}(v)$, and this holds in the integral form [7], for the case of $L^{\infty}$-weak solutions. Thus, by taking the $L^{\infty}$-norm along all characteristics, we have a bound on the time evolution of $\|\rho\|_{L^{\infty}}$

$$
\frac{d}{d t}\|\rho\|_{L^{\infty}} \leq\|\Delta K * \rho\|_{L^{\infty}}\|\rho\|_{L^{\infty}}
$$

In the case where $K$ is $C^{2}$, we immediately get that

$$
\|\Delta K * \rho\|_{L^{\infty}} \leq\|\Delta K\|_{L^{\infty}}\|\rho\|_{L^{1}}
$$


which is a priori bounded and thus by Grönwall's lemma, gives a global bound for $\|\rho\|_{L^{\infty}}$. Combining this with Theorem 3.3 provides the following result (the a priori bound has been proved in [47]):

Theorem 3.7 (Global-in-time solutions for $C^{2}$ potentials) Let $K$ be an admissible $C^{2}$ kernel. Then the weak solution of Theorem 3.3 exists for all time and we have a global in time bound

$$
\|\rho(\cdot, t)\|_{L^{\infty}} \leq e^{C t}\|\rho(\cdot, 0)\|_{L^{\infty}}
$$

where $C$ depends on $\|\Delta K\|_{L^{\infty}}$ and the mass of $\rho$.

We also obtain the following corollary of the previous section:

Corollary 3.8 (Infinite time blow-up for $C^{2}$ potentials) Let $K$ be an admissible $C^{2}$ kernel satisfying the conditions of Proposition 3.4. If the global-in-time weak solution of Theorem 3.7 has compact support, then it converges to a Dirac mass at the center of mass $c_{M}$ as $t \rightarrow \infty$.

Proof. The proof follows by applying Proposition 3.6 to the global solution, and noting that the solution $\tilde{R}$ of the ODE goes to zero as $t \rightarrow \infty$.

We now show that the same result holds for potentials satisfying the weaker Osgood condition

$$
\int_{0}^{1} \frac{1}{k^{\prime}(r)} d r=\infty
$$

Theorem 3.9 (Global-in time $L^{\infty}$ and infinite time blow-up for Osgood potentials [8]) Assume $k^{\prime \prime}(r)>0$ and that $k^{\prime}(r) / r$ monotone decreasing in $r$. Then on the interval of existence $\left(0, T^{*}\right)$

$$
\frac{d}{d t}\|\rho\|_{L^{\infty}}^{-1 / N} \geq-C(N, M) k^{\prime}\left(M^{1 / N}\|\rho\|_{L^{\infty}}^{-1 / N}\right)
$$

holds. As a consequence, if $K$ satisfies the Osgood condition (1.4) then for any compactly supported nonnegative $L^{\infty}$ solution of the aggregation equation stays bounded for all time and converges as $t \rightarrow \infty$ to a Dirac mass of size $M$ located at its center of mass $c_{M}$.

Proof. The proof involves an estimate for the characteristic lengthscale associated with a possible blowup. We would like to obtain a bound using only the $L^{\infty}$ and $L^{1}$ norms, the latter of which is conserved for nonnegative solutions. There is only one lengthscale that one can construct out of these norms, it is

$$
\delta=\left(\frac{M}{\|\rho\|_{L^{\infty}}}\right)^{1 / N}
$$

One obtains

$$
\dot{\delta}=-\frac{1}{N} M^{1 / N}\|\rho\|_{L^{\infty}}^{-1-1 / N} \frac{d}{d t}\|\rho\|_{L^{\infty}} \geq-\frac{1}{N} M^{1 / N}\|\rho\|_{L^{\infty}}^{-1 / N}\|\Delta K * \rho\|_{L^{\infty}}=-\frac{\delta}{N}\|\Delta K * \rho\|_{L^{\infty}} .
$$

In [8] a refined potential theory calculation proves that

$$
\|\Delta K * \rho\|_{L^{\infty}} \leq C M k^{\prime}(\delta) / \delta .
$$

The proof uses the fact that $k^{\prime}(r) / r$ is monotone decreasing so that $k^{\prime \prime}(r) / r-k^{\prime}(r) / r^{2} \leq 0$ away from the origin and thus $0<k^{\prime \prime}(r) \leq k^{\prime}(r) / r$ away from the origin. The upshot is that we have an a priori estimate on $\delta$, namely

$$
\dot{\delta} \geq-C(N, M) k^{\prime}(\delta) .
$$

If $k$ is Osgood, then $\delta$ can not go to zero in finite time, which in turn provides an a priori bound for $\|\rho\|_{L^{\infty}}$. 


\section{Shape of the blowup for $K=|x|$}

In this section, we explore the possibility of describing more in detail the finite-time blow-up proved in previous sections. With this purpose, we focus on finding certain blow-up self-similar solutions of (1.2) with homogeneous potentials. Suppose $K(x)$ is a potential with a Lipschitz point at the origin:

$$
K(x) \sim C|x| \text { as } x \rightarrow 0
$$

and suppose $\rho$ is a solution of (1.2) which blows up at $t=T^{*}$. We choose this special kernel for the following reasons: (1) kernels with Lipschitz points are one of the most common examples in the aggregation literature, (2) the special homogeneity of this kernel simplifies some of the analysis, and (3) when the blowup occurs at a point, it is only the local structure of the kernel at the origin that is important and moreover, to do similarity analysis we take it to be homogeneous. Close to the blow up time, one would expect $\rho$ to have small support (or at least to be highly concentrated). Therefore the velocity can be well approximated by

$$
v=-C \rho * \nabla|x| .
$$

From this remark, one would expect that the blow-up profile of (1.2) with a potential $K(x)$ satisfying (4.16) can be well approximated by the blow up profile of $(1.2)$ with $K(x)=C|x|$.

\subsection{One dimension}

In one space dimension, the kernel $K(x)=|x|$ reduces to Burgers equation via the formula $\psi(x, t)=\int_{0}^{x} \rho(x, t) d x$; one can easily see that $\psi$ satisfies a form of inviscid Burgers equation $[31,14]$. This gives families of exact solutions yielding finite-time blowup including the wellknown textbook linear-shock example which for $\rho$ gives an exact similarity solution

$$
\rho_{S}(x, t):=\frac{1}{R(t)} U\left(\frac{x}{R(t)}\right)
$$

where $U(x)$ is the uniform distribution on $[-1,1]$ and $R(t)=T^{*}-t$. This is an exact solution that concentrates mass at the blowup time. It is an example of a 'first kind' [5] similarity solution in which the timescale of the blowup (namely $R(t)$ ) can be predicted by dimensional analysis combined with mass conservation.

There is another class of exact similarity solutions for the Burgers blowup. They have the form

$$
\rho_{S}(x, t)=\frac{1}{(R(t))^{\alpha}} U\left(\frac{x}{(R(t))^{\beta}}\right), \quad R(t)=T^{*}-t,
$$

however in this case the similarity solution does not conserve mass. Dimensional analysis still implies $\alpha=1$, however $\beta$ is determined by a matching condition to the far field. The details of this calculation can be found in [31] with a local Taylor expansion result derived in [14], the upshot is that there is a global similarity solution for the Burgers blowup, that describes shock formation from generic odd initial data for Burgers, and hence blowup from even initial data for the aggregation problem. This exact solution has $\beta=3 / 2$ and there is a one parameter family of such solutions through a rescaling. This is an example of a 'second kind' similarity solution as in [5]. 


\subsection{On the non-existence of 'first kind' similarity in higher odd dimensions}

In [8] it was proved that first kind similarity solutions can not exist with support on open sets in any odd dimension larger than one.

The argument establishes that a first kind similiarity solution must satisfy

$$
v=-\lambda x
$$

for some constant $\lambda$, on the support of the solution. Using this fact, we arrive at the following nonexistence theorems for radially symmetric similarity solutions in odd dimensions $N$ higher than one.

Theorem 4.1 (Non-existence of $L^{p}$ similarity solutions [8]) Let $N$ be an odd space dimension larger than one and $K(x)=|x|$. Then there does not exist a nonnegative similarity solution in $L^{p}\left(\mathbb{R}^{N}\right)$ for $p>1$ whose support contains an open set.

Proof. The proof is elementary so we repeat it here. We start with the $3 \mathrm{D}$ case. Note that $\operatorname{div}(v)$ is a constant times the Newtonian potential convolved with $\rho$. Therefore, the distributional Laplacian of $\operatorname{div}(v)$ is a constant multiple of the $L^{p}$ function $\rho$. But from (4.17) it is clear that $\Delta(\operatorname{div}(v))=0$ on any open set on the support of $\rho$. Therefore $\rho$ is zero a.e. in any open set inside its support.

To extend this result from 3D to odd higher dimensions, we note that there is always some power of the Laplacian that can be applied to $\operatorname{div}(v)$ to obtain a constant times the identity functional, since the Newtonian potential is a constant multiple of $1 /|x|^{2-N}$ in $N$ dimensions. The rest of the argument follows in higher dimensions as well.

Theorem 4.2 (Non-existence of measure similarity solutions [8]) Let $N$ be an odd space dimension larger than one and $K(x)=|x|$. Then there do not exist any nonnegative nontrivial measure-valued similarity solutions, compactly supported on $\mathbb{R}^{N}$, whose support contains an open set.

Proof. The proof is similar to that of Theorem 4.1 except that we now consider $\rho$ to be a compactly supported measure. This means that $\nabla K * \rho$ and $1 /|x| * \rho$ can be understood in the sense of distributions (a distribution convolved with a distribution of compact support is a distribution). The distributional Laplacian of $\nabla \cdot v$ equals a constant times $\rho$ in the sense of distributions and due to (4.17) as above, the distributional Laplacian is zero on any open set contained in the support of $\rho$. Thus $\rho$ has no support on open sets.

Remark 4.3 (Even dimensions) Note that the argument fails in even dimensions because there is no local differential operator that inverts the convolution operator $1 /|x|$. Rather the appropriate operator is a non-local pseudo-differential operator.

\subsection{Numerical simulations of radial blowup in multiple dimensions, $K=|x|$}

Blowup in multiple dimensions has recently been studied numerically by Huang and the first author in [31]. We review those results in this subsection. The code uses a Lagrangian formulation of the problem and integrates the solution along characteristics, thereby eliminating numerical diffusion typically found in finite difference discretizations of Eulerian formulations of transport problems. Here we take advantage of the fact that our problem is a first order conservation law and thus we can use the method of characteristics to solve two coupled ODEs, one for the 
radial position $r$ and the other for the solution $\rho$. This method provides a natural 'adaptive grid scheme' to concentrate spatial resolution near the blowup point or set, and was employed to investigate gravitational collapse by Brenner and Witelski [17]. Moreover, for nonnegative initial data, we have the monotonicity condition $\frac{\partial}{\partial r} K * \rho \geq 0, \Delta_{r} K * \rho>0$, i.e the points always move towards to the origin and the magnitude is always increasing. Thus our scheme preserves the positivity of the solution. The numerical results indicate that this simple scheme resolves the profiles quite well, both near the core and far away from it. If the self-similarity were first kind, then the characteristics would exactly preserve the spatial resolution going into the blowup. For a second-kind similarity solution with anomalous scaling (i.e. the characteristics do not scale in time as the similarity variable) we loose resolution over time, however at a relatively slow rate compared with the dynamics of blowup. In radial coordinates, the original equation can be written as

$$
\rho_{t}=\frac{\partial \rho}{\partial r} \frac{\partial}{\partial r} K * \rho+\rho \Delta_{r} K * \rho
$$

where $\Delta_{r}=\partial_{r r}+\frac{N-1}{r} \partial_{r}$. The system of ODEs along the characteristics is thus

$$
\frac{d r}{d t}=-\frac{\partial}{\partial r} K * \rho, \quad \frac{d \rho}{d t}=\rho \Delta_{r} K * \rho
$$

Instead of calculating $K * \rho$ once and taking the numerical derivatives to solve (4.19), we find $\frac{\partial}{\partial r} K * \rho$ and $\Delta_{r} K * \rho$ directly by computing the derivatives of the kernel, i.e

$$
\begin{array}{r}
\frac{\partial}{\partial r} K * \rho=c_{N} \int_{0}^{\infty} \rho\left(r^{\prime}\right) r^{\prime N-1} \int_{0}^{\pi} \frac{r-r^{\prime} \cos \theta}{\sqrt{r^{2}+r^{\prime 2}-2 r r^{\prime} \cos \theta}} \sin ^{N-2} \theta d \theta d r^{\prime}, \\
\Delta_{r} K * \rho=(N-1) c_{N} \int_{0}^{\infty} u\left(r^{\prime}\right) r^{\prime n-1} \int_{0}^{\pi} \frac{1}{\sqrt{r^{2}+r^{\prime 2}-2 r r^{\prime} \cos \theta}} \sin ^{N-2} \theta d \theta d r^{\prime},
\end{array}
$$

where $c_{N}$ is an constant arising from integration of angular variables. The computation can still be expensive, because at each point we have to perform a double integration. The expense can be reduced by observing the homogeneity of the kernel, which gives the following formulation

$$
\begin{gathered}
\int_{0}^{\pi} \frac{r-r^{\prime} \cos \theta}{\sqrt{r^{2}+r^{\prime 2}-2 r r^{\prime} \cos \theta}} \sin ^{N-2} \theta d \theta=\left\{\begin{array}{ll}
\int_{0}^{\pi} \frac{(1-\tilde{\rho} \cos \theta) \sin ^{N-2} \theta}{\sqrt{1+\tilde{\rho}^{2}-2 \tilde{\rho} \cos \theta}} d \theta, & \text { if } r^{\prime} \leq r \\
\int_{0}^{\pi} \frac{(\tilde{\rho}-\cos \theta) \sin ^{N-2} \theta}{\sqrt{1+\tilde{\rho}^{2}-2 \tilde{\rho} \cos \theta}} d \theta, & \text { if } r^{\prime} \geq r
\end{array}, \sin ^{N-2} \theta d \theta=\frac{1}{\max \left(r, r^{\prime}\right)} \int_{0}^{\pi} \frac{1}{\sqrt{1+\tilde{\rho}^{2}-2 \tilde{\rho} \cos \theta}} \sin ^{N-2} \theta d \theta,\right.
\end{gathered}
$$

where $\tilde{\rho}=\min \left(r, r^{\prime}\right) / \max \left(r, r^{\prime}\right)$. In this way, the integrations of the kernel with respect to the angular variable have only to be calculated once at the very beginning as functions of $\tilde{\rho} \in[0,1]$, i.e we only need to perform numerical integrations once for the auxiliary functions

$$
\begin{gathered}
I_{1}(\tilde{\rho})=\int_{0}^{\pi} \frac{(1-\tilde{\rho} \cos \theta) \sin ^{N-2} \theta}{\sqrt{1+\tilde{\rho}^{2}-2 \tilde{\rho} \cos \theta}} d \theta, \quad I_{2}(\tilde{\rho})=\int_{0}^{\pi} \frac{(\tilde{\rho}-\cos \theta) \sin ^{N-2} \theta}{\sqrt{1+\tilde{\rho}^{2}-2 \tilde{\rho} \cos \theta}} d \theta, \\
I_{3}(\tilde{\rho})=\int_{0}^{\pi} \frac{\sin ^{N-2} \theta}{\sqrt{1+\tilde{\rho}^{2}-2 \tilde{\rho} \cos \theta}} d \theta .
\end{gathered}
$$

In odd dimension, using the fact that the successive derivative of the kernel $K(x)=|x|$ is proportional to the fundamental solution of the Laplace equation, one can further reduce the computation to be $O\left(N_{1}\right)$ per time step, where $N_{1}$ is the number of grid points. 

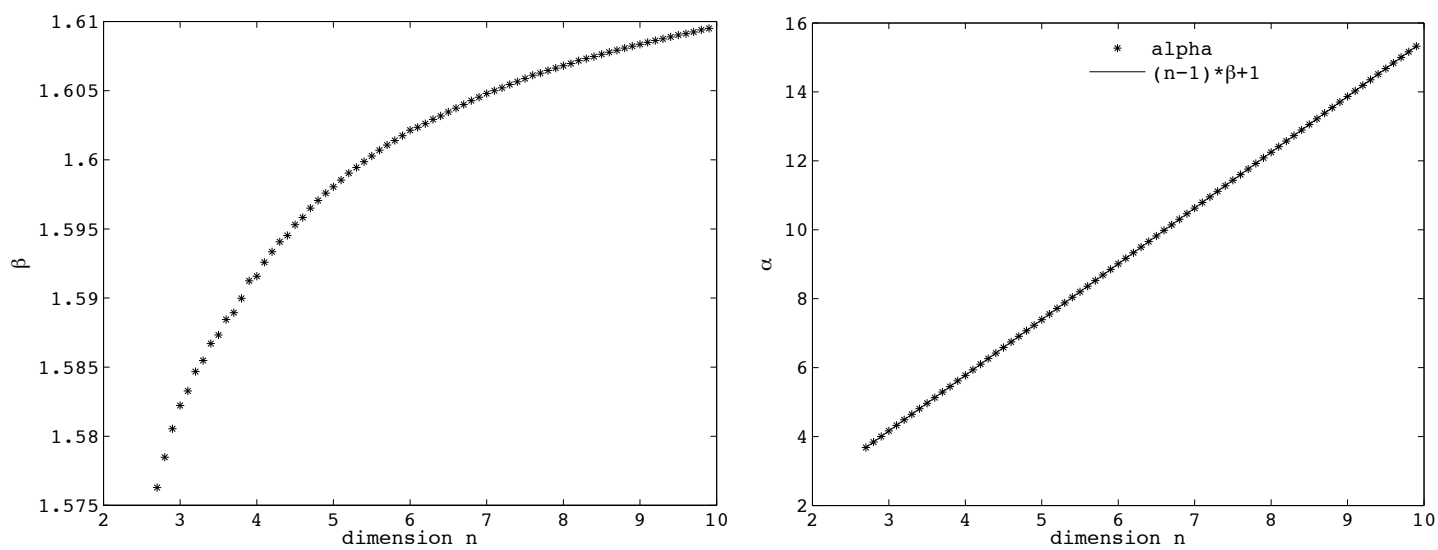

Figure 1: The exponents characterizing the blowup in different spatial dimensions: $\beta($ Left) and $\alpha$ (right) [31]. The comparison of the estimated $\alpha$ is in perfect agreement with dimensional analysis.

The computations are performed, tracking the blowup over many orders of magnitude, for example with $\rho$ well-resolved up to $10^{40}$ or higher. Exponents $\alpha$ and $\beta$ are estimated from the numerical data and shown in Fig. 1. The graph on the right clearly shows $\alpha=\beta(N-1)+1$ as predicted from dimensional analysis, however conservation of mass, which would give $\alpha=N \beta$ is not satisfied. The values of $\beta$ shown in Fig. 1 on the left reveal a second kind similarity solution, where the initial blowup does not concentrate mass, but instead exhibits a power-law behavior at the origin. At the blowup time, the profile behaves as $\rho\left(x, T^{*}\right)=|x|^{-\beta_{1}}$ where $\beta_{1}=\alpha / \beta$. We conjecture that the solution concentrates mass immediately after the initial blowup time and that these blowup solutions for higher dimensions are analogues of the $\beta=3 / 2$ second kind similarity solution for the Burgers singularity in one space dimension. Note that this power law blowup implies that $\| \rho\left(\cdot, t \|_{L^{p}} \rightarrow \infty\right.$ for all $p \geq N / \beta_{1}=1+\frac{1+1 / \beta}{N-1+1 / \beta}$. This is larger than the rigorous theory for $L^{p}$ blowup discussed in Section 6, and thus consistent with what is proven in that section.

\section{$5 \quad$ Well-posedness theory for measure solutions}

In order to study the porous media equation and the Fokker-Plank equation, Otto [45, 32] (also in collaboration with Jordan and Kinderlleher) introduced a formal Riemannian structure in the space $\mathcal{P}_{2}\left(\mathbb{R}^{N}\right)$ of probability measures of finite quadratic moment. The geodesic distance induced by this Riemannian structure is the Wasserstein distance (see $[3,50,51]$ for a definition of the Wasserstein distance). The Fokker-Plank equation

$$
\partial_{t} u-\operatorname{div}(\nabla u+u \nabla V)=0
$$

and the porous media equation

$$
\partial_{t} u=\Delta\left(u^{m}\right)
$$

can be interpreted as gradient flows in this Riemannian structure for the energy

$$
E(u)=\int_{\mathbb{R}^{N}} u(\log (u)+V) d x \quad \text { and } \quad E(u)=\frac{1}{m-1} \int_{\mathbb{R}^{N}} u^{m} d x \quad \text { respectively. }
$$


In the same spirit, the aggregation equation can be interpreted as a gradient flow in this Riemannian structure for the interaction energy

$$
E_{K}(\mu)=\frac{1}{2} \iint K(x-y) d \mu(x) d \mu(y)
$$

In the next subsection, we give a brief description of this Riemannian structure and we explain why, formally, the aggregation equation can be thought as a gradient flow of the interaction energy with respect to this Riemannian structure. In the second subsection, we will state the rigorous results which were obtained in [20] using this point of view: in particular a well-posedness theory for measure solutions is developped. A concept of measure solution for the aggregation equation is important since it allows us to continue the solution after the initial $L^{p}$-blowup discribed in the previous sections.

\subsection{The aggregation equation is a gradient flow of the interaction energy with respect to the Wasserstein distance}

In this subsection, we give a very brief, intuitive and nonrigorous explanation of what is a gradient flow with respect to the Wasserstein distance. For a complete understanding of this theory we refer the reader to [3]. Let us first describe the Riemannian structure underlying the Wasserstein distance. To every point $\mu \in \mathcal{P}_{2}\left(\mathbb{R}^{N}\right)$ is associated the tangent space

$$
\operatorname{Tan}_{\mu} \mathcal{P}_{2}\left(\mathbb{R}^{N}\right):=\text { completion of }\left\{\nabla \phi: \phi \in C_{c}^{\infty}\left(\mathbb{R}^{N}\right)\right\} \text { in } L^{2}\left(\mu, \mathbb{R}^{N}\right)
$$

endowed with the inner product

$$
\langle\vec{v}, \vec{w}\rangle_{\mu}:=\int_{\mathbb{R}^{N}} \vec{v}(x) \cdot \vec{w}(x) d \mu(x)=\langle\vec{v}, \vec{w}\rangle_{L^{2}\left(\mu, \mathbb{R}^{N}\right)} .
$$

The elements of the tangent space are vector fields. Roughly speaking, these vector fields push probability measures on the manifold. To be a little more precise, a vector field $\vec{w}: \mathbb{R}^{N} \rightarrow \mathbb{R}^{N}$ is said to be tangent to a curve of probability measure $\left(\mu_{t}\right)_{t \in(0, T)}$ at time $t_{0}$ if there exists a family of vector fields $\left(\vec{v}_{t}\right)_{t \in(0, T)}$ such that

$$
\begin{gathered}
\vec{v}_{t} \in \operatorname{Tan}_{\mu_{t}} \mathcal{P}_{2}\left(\mathbb{R}^{N}\right), \\
\partial_{t} \mu_{t}+\operatorname{div}\left(\mu_{t} \vec{v}_{t}\right)=0, \\
\vec{v}_{t_{0}}=\vec{w} .
\end{gathered}
$$

At time $t_{0}$ the probability measure $\mu_{t_{0}}$ is "pushed" by the vector field $\vec{w}$ according to the continuity equation (5.26). Given a curve of probability measures which is absolutely continuous with respect to the metric induced by the Wasserstein distance, it is known (see for example [3]) that there exists a unique tangent vector to this curve for almost every $t$.

We say that an absolutely continuous curve of probability measure $\left(\mu_{t}\right)_{t \in(0, T)}$ is a gradient flow for the interaction energy (5.24) if at almost every $t$, the tangent vector to this curve points in the direction which decreases the interaction energy the fastest. So let us ask the following question: given a fixed probability measure $\mu$, what is the element of the tangent space $\operatorname{Tan}_{\mu} \mathcal{P}_{2}\left(\mathbb{R}^{N}\right)$ which point in the direction which decreases the interaction energy the fastest? In other words, if one were to push the density $\mu$ according to some vector field $\vec{w}$ what would be the best vector field

to choose in order to decrease the interaction energy the fastest? To do this, let us compute the 
derivative of the energy along a curve $\left(\nu_{t}\right)_{t \in(-\epsilon, \epsilon)}$ starting at $\mu$ and being pushed according to the continuity equation by the vector field $\vec{w}$, i.e.:

$$
\left\{\begin{array}{l}
\partial_{t} \nu_{t}+\operatorname{div}\left(\nu_{t} \vec{w}\right)=0, \quad t \in(-\epsilon, \epsilon) \\
\nu_{0}=\mu
\end{array}\right.
$$

For small $t, \nu_{t}$ is a small displacement of the probability measure $\mu$ in the direction $\vec{w}$.

$$
\begin{aligned}
\frac{d}{d t} E\left(\nu_{t}\right) & =\frac{d}{d t} \frac{1}{2} \iint K(x-y) \nu_{t}(x) \nu_{t}(y) d x d y & & \\
& =\iint K(x-y) \nu_{t}(y) \partial_{t} \nu_{t}(x) d x d y & & \text { by symmetry of } K \\
& =-\iint K(x-y) \nu_{t}(y) \operatorname{div}\left(\nu_{t} \vec{w}\right) d x d y & & \text { by }(5.28) \\
& =-\int\left(K * \nu_{t}\right) \operatorname{div}\left(\nu_{t} \vec{w}\right) d x & & \\
& =\int\left(\nabla K * \nu_{t}\right) \cdot \vec{w} d \nu_{t}(x) . & & \text { by integration by parts }
\end{aligned}
$$

Evaluating at $t=0$ we find

$$
\left.\frac{d}{d t} E\left(\nu_{t}\right)\right|_{t=0}=\int(\nabla K * \mu) \cdot \vec{w} d \mu(x) .
$$

It is then clear that, up to multiplication by a scalar, the optimal $\vec{w}$ to choose in (5.29) in order to decrease the interaction energy the fastest is $\vec{w}=-\nabla K * \mu$.

So, a curve of probability measure $\left(\mu_{t}\right)_{t \in(0, T)}$ is a gradient flow of the interaction energy if at almost every $t$ the tangent vector to this curve is $-\nabla K * \mu_{t}$. Given the definition of a tangent vector to a curve in $\mathcal{P}_{2}\left(\mathbb{R}^{N}\right)$, this can be rephrased as follows: a curve of probability measure $\left(\mu_{t}\right)_{t \in(0, T)}$ is a gradient flow of the interaction energy if at almost every $t$, the probality measure $\mu_{t}$ is pushed according to the continuity equation in the direction $\vec{w}=-\nabla K * \mu_{t}$, i.e. $\left(\mu_{t}\right)_{t \in(0, T)}$ must satisfies the aggregation equation.

\subsection{Rigorous results obtained using the gradient flow structure of the aggre- gation equation}

The approach of [3] in proving the existence of an absolutely continuous curve of probability measure satisfying the gradient flow problem is based on a variational version of the implicit Euler scheme, sometimes referred to as the Jordan-Kinderlehrer-Otto (JKO) scheme or minimizing movement scheme $[32,1,3]$. Given an initial measure $\mu_{0} \in \mathcal{P}_{2}$ and time-step $\tau>0$, we consider a sequence $\mu_{k}^{\tau}$ recursively defined by $\mu_{0}^{\tau}=\mu_{0}$ and

$$
\mu_{k+1}^{\tau} \in \arg \min _{\mu \in \mathcal{P}_{2}}\left\{E[\mu]+\frac{1}{2 \tau} d_{W}^{2}\left(\mu_{k}^{\tau}, \mu\right)\right\}
$$

for $k=0,1,2, \ldots$ Here $d_{W}^{2}\left(\mu_{k}^{\tau}, \mu\right)$ denotes the square of the Wasserstein distance between the probability measures $\mu_{k}^{\tau}$ and $\mu$.

In the case where $E$ is the interaction energy, the well-posedness of definition (5.30) and the convergence of $\mu_{k}^{\tau}$ as $\tau \rightarrow 0$ (after a suitable interpolation) to a limit which satisfies a weak 
formulation of the aggregation equation is established in [20]. In this work, we require the potential $K$ to be $\lambda$-convex for some $\lambda \in \mathbb{R}$. This means that there must exists a $\lambda \in \mathbb{R}$ such that:

$$
K(x)-\frac{\lambda}{2}|x|^{2} \quad \text { is convex. }
$$

Most potentials of interest are $\lambda$-convex. For example a pointy potential such as $K(x)=1-e^{-|x|}$ is $(-1)$-convex. Here is the main theorem:

Theorem 5.1 (Existence of measure solutions [20]) Given any $\mu_{0} \in \mathcal{P}_{2}\left(\mathbb{R}^{d}\right)$, then there exists an absolutely continuous curve $\left(\mu_{t}\right)_{t \in[0,+\infty)}$ satisfying

$$
\begin{aligned}
& \partial_{t} \mu_{t}+\operatorname{div}\left(\mu_{t} \vec{v}_{t}\right)=0 \text { in } \mathcal{D}^{\prime}\left([0, \infty) \times \mathbb{R}^{d}\right), \\
& \vec{v}_{t}=-\partial^{0} K * \mu_{t},
\end{aligned}
$$

with $\mu_{\{t=0\}}=\mu_{0}$. Moreover, the energy identity

$$
E\left[\mu_{t_{b}}\right]-E\left[\mu_{t_{a}}\right]=-\int_{t_{a}}^{t_{b}}\left\|v_{t}\right\|_{L^{2}\left(\mu_{t}, \mathbb{R}^{N}\right)} d t
$$

holds for all $0 \leq t_{a} \leq t_{b}<\infty$.

Here $\partial^{0} K$ is the unique element of minimal norm in the subdifferential of $K$. Simply speaking, since $K$ is smooth away from the origin and radially symetric, we have $\partial^{0} K(x)=\nabla K(x)$ for $x \neq 0$ and $\partial^{0} K(0)=0$. Note that $\mu_{t}$ being a measure it is important for $\partial^{0} K$ to be defined for every $x \in \mathbb{R}^{N}$ for (5.32) to make sense. We also have:

$$
\left(\partial^{0} K * \mu\right)(x)=\int_{y \neq x} \nabla K(x-y) d \mu(y) .
$$

Remark 5.2 Such a problem has been widely studied for smooth convex potentials in [3], where convergence of the discrete scheme to a suitable limit is shown. However, allowing for $K(x)$ to have a Lipschitz singularity at the origin (e.g. $K(x)=1-e^{-|x|}$ ) requires some improvements of the arguments in [3, Part I]. Simplistically speaking, [3, Part I] provide a theory for weak measure solutions for potentials which do not produce blow-up in finite time whereas [20] provides a theory for potentials which produce blow-up.

One of the key properties of the constructed solutions is the stability with respect to the Wasserstein distance $d_{W}$ :

Theorem 5.3 (Uniqueness and $d_{W}$-Contraction [20]) Given two gradient flow solutions $\left(\mu_{t}^{1}\right)_{t \in[0,+\infty)}$ and $\left(\mu_{t}^{2}\right)_{t \in[0,+\infty)}$ in the sense of the theorem above, we have

$$
d_{W}\left(\mu_{t}^{1}, \mu_{t}^{2}\right) \leq e^{-\lambda t} d_{W}\left(\mu_{0}^{1}, \mu_{0}^{2}\right) \quad \forall t \geq 0
$$

where $\lambda \in \mathbb{R}$ is the constant of $\lambda$-convexity of the potential $K$. In particular, we have a unique gradient flow solution for any given $\mu_{0} \in \mathcal{P}_{2}\left(\mathbb{R}^{d}\right)$.

This stability result is not only useful for showing uniqueness but it is mainly a tool for approximating general solutions by particle ones: given an initial condition $\mu_{0}$ one can approximate it by a finite number of particles (i.e. dirac delta functions). By increasing the number of particles this approximation can be made as accurate as we want with respect to the Wasserstein distance. The particles approximation obeys the system of ODE's (2.6) described earlier. 
We have seen in Theorem 2.1 that if the potential does not satisfy the Osgood condition this system of ODE collapse on itself in finite time. Moreover Theorem 2.1 provides an upperbound for the collapse time which is independent of the number of particles. Therefore by increasing the number of particles and by making the approximation more and more accurate one doesn't change this upperbound on the collapse time. This shows that the upperbound on the collapse time holds for the full solution:

Theorem 5.4 (Finite Time Total Collapse [20]) Assume that the potential $K(x)=k(|x|)$ satisfies $\int_{0}^{1} \frac{d r}{k^{\prime}(r)}<+\infty$ and that $k^{\prime \prime}(r)$ is monotone in some neighborhood $(0, \delta)$ of the origin. Let $\left(\mu_{t}\right)_{t \in[0,+\infty)}$ denote the unique gradient flow solution starting from the probability measure $\mu_{0}$ with center of mass

$$
x_{c}:=\int_{\mathbb{R}^{d}} x d \mu_{0},
$$

supported in $B\left(x_{c}, R_{0}\right)$. Then there exists $T^{*}$, depending only on $R_{0}$, such that $\mu_{t}=\delta_{x_{c}}$ for all $t \geq T^{*}$.

In [20], the same strategy of proving results at the ODE level and then extending them to the full measure solution using the stability Theorem 5.3 is used to prove confinement results for potentials which are repulsive in the short range and attractive in the long range. In this kind of problems one derive necessary conditions on the strength of attraction versus the strength of repulsion of the potential so that solutions stay in a ball of finite radius for all times.

\section{$6 \quad L^{p}$ theory and instantaneous mass concentration}

As we have seen in the previous section, one of the distinctive feature of the aggregation equation with non-Osgood potential is that, starting from initial data which are absolutely continuous with respect to the Lebesgue measure, it can generate mass concentration in finite time - in which case the solution is no longer absolutely continuous with respect to the Lebesgue measure. From the local existence theory developed in [7], and reviewed in section 3 of this paper, it is clear that solutions starting with $L^{1} \cap L^{\infty}$-initial data can not concentrate mass instantaneously, i.e. there is a least a short time interval during which the solution stay absolutely continuous with respect to the Lebesgue measure. A natural question to ask is how regular need be the initial data in order to guarantee that the solution will not concentrate mass instantaneously? In [10], we show that if the initial data belongs to $L^{1} \cap L^{p}$ for $p>N / N-1$ then solution remains in $L^{p} \cap L^{1}$ for some finite time. On the other hand, we believe that one can construct examples for which $p \leq N / N-1$ and the initial data in $L^{1} \cap L^{p}$ yet the solution concentrates mass instantaneously. This suggests a critical $L^{p}$ space for which one can achieve instantaneous mass concentration. This section summarizes recent existence theory for $L^{p}$-data [10].

Theorem 6.1 (Local existence [10]) Suppose $\nabla K \in W^{1, q}\left(\mathbb{R}^{N}\right)$ and suppose $u_{0} \in L^{p}\left(\mathbb{R}^{N}\right)$ is nonnegative ( $p$ and $q$ are Hölder conjugates). Then there exists a time $T^{*}>0$ and a nonnegative function $\rho \in C\left(\left[0, T^{*}\right], L^{p}\left(\mathbb{R}^{N}\right)\right) \cap C^{1}\left(\left[0, T^{*}\right], W^{-1, p}\left(\mathbb{R}^{N}\right)\right)$ such that

$$
\begin{array}{cc}
\rho^{\prime}(t)+\operatorname{div}[\rho(t) v(t)]=0 & \forall t \in\left[0, T^{*}\right] \\
v(t)=-\rho(t) * \nabla K & \forall t \in\left[0, T^{*}\right] \\
\rho(0)=\rho_{0} &
\end{array}
$$

Moreover the solution can be continued up to a time $T_{\max } \in(0,+\infty]$, and If $T_{\max }<+\infty$, then $\lim _{t \rightarrow T_{\max }} \sup _{\tau \in[0, t]}\|\rho(\tau)\|_{L^{p}}=+\infty$. 
To prove this theorem, we use the duality between $L^{p}$ and $L^{q}$, which guaranties enough smoothness in the velocity field $\vec{v}=\nabla K * \rho$ to define characteristics. Existence of a solution is proved using characteristics, as in [52] for 2D incompressible Euler flows and as in [7] for the $L^{\infty}$ theory for the aggregation problem.

Proving uniqueness for solution which solely belongs to $L^{p}\left(\mathbb{R}^{N}\right)$ is challenging. Instead, we consider solutions in $\mathcal{P}_{2}\left(\mathbb{R}^{N}\right) \cap L^{p}\left(\mathbb{R}^{N}\right)$, where $\mathcal{P}_{2}\left(\mathbb{R}^{N}\right)$ denotes the space of probability measure with finite second moment [10]. Note that $\mathcal{P}_{2}\left(\mathbb{R}^{N}\right) \cap L^{p}\left(\mathbb{R}^{N}\right)$ can also be thought as the space of nonnegative functions which belong to $L^{1} \cap L^{p}$ and whose second moment are bounded. We first prove that if the initial data is in $\mathcal{P}_{2} \cap L^{p}$ then the solution stay in $\mathcal{P}_{2} \cap L^{p}$ as long as it exists. We then prove, using an argument based on optimal transport theory, first introduced by Lopers in [39] and then used by Carrillo and Rosado in [23], that such solutions are unique:

Theorem 6.2 (Uniqueness of $L^{p}$ solutions with bounded second moment [10]) Let $\rho_{1}$, $\rho_{2}$ be two solutions of equation (6.33) in the interval $\left[0, T^{*}\right]$ with initial data $\rho_{0} \in \mathcal{P}_{2} \cap L^{p}\left(\mathbb{R}^{N}\right)$, $1<p<\infty$ and assume that $v$ is given by $v=-\nabla K * \rho$, with $K$ such that $\nabla K \in W^{1, q}\left(\mathbb{R}^{N}\right), p$ and $q$ conjugates. Then $\rho_{1}(t)=\rho_{2}(t)$ for all $0 \leq t \leq T^{*}$.

As in the $L^{1} \cap L^{\infty}$-theory, the Osgood criteria play an important role to determine wether or not the aggregation equation is globally well posed. In [10] we have weakened the hypothesis needed on monotonicity of the potential $\mathrm{K}$ in order for the Osgood criteria to be relevant. To be more precise, we we consider the class of natural potentials. A potential is said to be natural if

a) it is radially symmetric, i.e.: $K(x)=k(|x|)$,

b) it is smooth away from the origin and it's singularity at the origin is not worth than Lipshitz,

c) it does not exhibit pathological oscillation at the origin, and

d) its derivatives decays fast enough at infinity.

These conditions are more rigorously stated in [10]. Moreover a natural potential is said to be repulsive in the short range if it has a local max at the origin and it is said to be strictly attractive in the short range if it has a strict local min at the origin.

Remark 6.3 The gradient of natural potentials automatically belongs to $W^{1, q}$ for $q<N$, therefore we have local existence and uniqness in $L^{p} \cap \mathcal{P}_{2}, p>\frac{N}{N-1}$.

Theorem 6.4 (Osgood condition for global well posedness [10]) Suppose $K$ is a natural potential.

(i) If $K$ is repulsive in the short range, then the aggregation equation is globally well posed in $\mathcal{P}_{2}\left(\mathbb{R}^{N}\right) \cap L^{p}\left(\mathbb{R}^{N}\right), p>N /(N-1)$.

(ii) If $K$ is strictly attractive in the short range, the aggregation equation is globally well posed in $\mathcal{P}_{2}\left(\mathbb{R}^{N}\right) \cap L^{p}\left(\mathbb{R}^{N}\right), p>N /(N-1)$, if and only if the Osgood condition (1.4) is satisfied.

By globally well posed in $\mathcal{P}_{2}\left(\mathbb{R}^{N}\right) \cap L^{p}\left(\mathbb{R}^{N}\right)$, we mean that a solution of the aggregation equation which start in $\mathcal{P}_{2}\left(\mathbb{R}^{N}\right) \cap L^{p}\left(\mathbb{R}^{N}\right)$ will stay in $\mathcal{P}_{2}\left(\mathbb{R}^{N}\right) \cap L^{p}\left(\mathbb{R}^{N}\right)$ for all time. If the equation is not globally well posed, the $L^{p}$-norm of the solution will blowup in finite time. In the above discussion, the bound $p>N /(N-1)$ holds for a Lipschitz continuous potential $K$. For smoother potentials, the bound could be made sharper - the necessary condition being that characteristics of the velocity field are well-defined. 


\section{Conclusions}

This review article summarizes a series of recent definitive results on the well-posedness of aggregation equations in multiple space dimensions. For the case of bounded data, we find that the Osgood condition (1.4) provides a necessary and sufficient condition on an attractive potential such that the solution exists for all time. For the case of measure-valued data, unique solutions exist for all time even when the Osgood condition is violated, however there is information loss in the case of attractive potentials that violate the Osgood condition, which can be seen in the fact that the solution collapses to a point in finite time. When bounded solutions blowup in finite time, they are observed in numerical simulations to exhibit second kind scaling in which the initial blowup does not concentrate mass but rather involves a weaker singularity that remains in $L^{p}$ for some range of $p$. This suggests that one should study well-posedness of solutions in the $L^{p}$-spaces, which we consider for those values of $p$ that give well-defined characteristics. In the case of $L^{p}$ initial data, we prove local-well posedness of the problem provided $p>p_{c}$, where $p_{c}$ is determined by the regularity of the kernel at the origin. For $p$ smaller than the critical $p$, one can construct examples in which the solution instantaneously concentrates mass, thereby suggesting that bound on $p$ for which the $L^{p}$ problem is locally well-posed, is sharp. One such example is the case where $k(x)=|x|$ and the initial density $\rho$ is radially symmetric with a power law behavior $\rho \sim|x|_{1}^{\beta}$ at the origin, as in the case of the numerical solution described in section 4 .

\section{Acknowledgments.-}

This paper is dedicated to Andrew Majda on the occasion of his 60th Birthday. The authors would like to thank our collaborators on the works cited here. They include Jeremy Brandman, José Carrillo, Marco DiFrancesco, Alessio Figalli, Yanghong Huang, Jesus Rosado, and Dejan Slepcev. We also acknowledge the office of Naval Research, the Army Research Office, and NSF grant DMS-0907931. TL thanks Fudan University for their hospitality during the conference in honor of Andrew Majda's 60th birthday.

\section{References}

[1] M. Aguen, Existence of solutions to degenerate parabolic equations via the MongeKantorovich theory, Adv. Differential Equations., 10, No 3 (2005), pp. 309-360.

[2] L. Ambrosio and P. Bernard, Uniqueness of signed measures solving the continuity equation for Osgood vector fields, Rendiconti Lincei-Mathematica e Applicazioni, 19(3), (2008), pp. 237-245.

[3] L.A. Ambrosio, N. Gigli, and G. Savaré, Gradient flows in metric spaces and in the space of probability measures, Lectures in Mathematics, Birkhäuser, 2005.

[4] R.P. Agarwal and V. Lakshmikantham, Uniqueness and Nonuniqueness Criteria for Ordinary Differential Equations, Series in Real Analysis, 6, World Scientific Publishing Co., Inc., River Edge, New York, 1993.

[5] G. I. Barenblatt, Scaling, self-similarity, and intermediate asymptotics, Cambridge Univ. Press, 1996.

[6] D. Benedetto, E. Caglioti, M. Pulvirenti, A kinetic equation for granular media, RAIRO Modél. Math. Anal. Numér., 31, (1997), 615-641. 
[7] A.L. Bertozzi and J. Brandman, Finite-time blow-up of $L^{\infty}$-weak solutions of an aggregation equation, to appear in Comm. Math. Sci.

[8] A. L. Bertozzi, J. A. Carrillo, and T. Laurent, Blowup in multidimensional aggregation equations with mildly singular interaction kernels, Nonlinearity, 22 (2009) pp. 683-710.

[9] A.L. Bertozzi And T. Laurent, Finite-time Blow-up of Solutions of an Aggregation Equation in $\mathbb{R}^{n}$, Comm. Math. Phys., 274 (2007), pp. 717-735.

[10] A. L. Bertozzi, T. Laurent, and J. Rosado, $L^{p}$ Theory for the Aggregation Equation, manuscript in preparation, 2009.

[11] P. Biler And A. Woyczyński Global and Expoding Solutions for Nonlocal Quadratic Evolution Problems, SIAM J. Appl. Math., 59 (1998), pp. 845-869.

[12] A. Blanchet, J. A. Carrillo, and N. Masmoudi, Infinite Time Aggregation for the Critical Patlak-Keller-Segel model in $\mathbb{R}^{2}$, to appear in Comm. Pure Appl. Math.

[13] A. Blanchet, J. Dolbeault, and B. Perthame, Two-dimensional Keller-Segel model: optimal critical mass and qualitative properties of the solutions, Electron. J. Differential Equations, (2006), No. 44, 32 pp. (electronic).

[14] M. Bodnar and J.J.L. VelázQuez, An integro-differential equation arising as a limit of individual cell-based models, J. Differential Equations 222, (2006), pp. 341-380.

[15] S. Boi, V. Capasso And D. Morale, Modeling the aggregative behavior of ants of the species Polyergus rufescens, Spatial heterogeneity in ecological models (Alcalá de Henares, 1998), Nonlinear Anal. Real World Appl., 1 (2000), pp. 163-176.

[16] M.P. Brenner, P. Constantin, L.P. Kadanoff, A. Schenkel and S.C. VenkataraMANI, Diffusion, attraction and collapse, Nonlinearity 12, (1999), pp. 1071-1098.

[17] Michael P. Brenner and Thomas P. Witelski On spherically symmetric gravitational collapse. J. Statist. Phys., (1998) 93(3-4):863899.

[18] M. Burger, V. Capasso And D. Morale, On an aggregation model with long and short range interactions, Nonlinear Analysis. Real World Applications. An International Multidisciplinary Journal, 8 (2007), pp. 939-958.

[19] M. Burger And M. Di Francesco, Large time behavior of nonlocal aggregation models with nonlinear diffusion, Networks and Heterogenous Media, 3(4), (2008), pp. 749-785.

[20] J. A. Carrillo, M. Difrancesco, A. Figalli, T. Laurent and D. Slepcev Global-intime weak measure solutions, finite-time aggregation and confinement for nonlocal interaction equations, preprint 2009.

[21] J.A. Carrillo, R.J. McCann, and C. Villani, Kinetic equilibration rates for granular media and related equations: entropy dissipation and mass transportation estimates, Rev. Matemática Iberoamericana, 19 (2003), pp. 1-48.

[22] J.A. Carrillo, R.J. McCann, and C. Villani, Contractions in the 2-Wasserstein length space and thermalization of granular media, Arch. Ration. Mech. Anal. 179 (2006), pp. 217263. 
[23] J.A. Carrillo, J. Rosado Uniqueness of bounded solutions to Aggregation equations by optimal transport methods, preprint.

[24] Y.-L. Chunang, Y. R. Huang, M. R. D’Orsogna and A. L. Bertozzi, Multi-vehicle flocking: scalability of cooperative control algorithms using pairwise potentials, IEEE International Conference on Robotics and Automation, 2007, pp. 2292-2299.

[25] P. Constantin, A. Majda, And E. Tabak, Formation of strong fronts in the 2D quasigeostrophic thermal active scalar, Nonlinearity, 7 (1994), pp. 1495-1533.

[26] J. Dolbeault and B. Perthame, Optimal critical mass in the two-dimensional KellerSegel model in $\mathbb{R}^{2}$, C. R. Math. Acad. Sci. Paris, 339 (2004), pp. 611-616.

[27] Q. Du And P. Zhang, Existence of Weak Solutions to Some Vortex Density Models, SIAM J. Math. Anal., 34(6), (2003), pp. 1279-1299.

[28] V. Gazi And K. Passino, Stability analysis of swarms, IEEE Trans. Auto. Control, 48 (2003), pp. 692-697.

[29] D. Holm And V. PutKaradze, Formation of clumps and patches in self-aggregation of finite-size particles, Physica D 220, (2006), pp. 183-196.

[30] D. Holm and V. Putkaradze, Aggregation of finite size particles with variable mobility, Phys. Rev. Lett., (2005) 95:226106.

[31] Y. HuAng And A. L. Bertozzi, Self-similar blowup solutions to an aggregation equation, preprint 2009.

[32] R. Jordan, D. Kinderlehrer, And F. Otto, The variational formulation of the FokkerPlanck equation, SIAM J. Math. Anal., 29 (1998), pp. 1-17.

[33] E.F. Keller AND L.A. SEgel, Initiation of slide mold aggregation viewed as an instability, J. Theor. Biol., 26 (1970).

[34] T. Laurent, Local and Global Existence for an Aggregation Equation, Communications in Partial Differential Equations, 32 (2007), pp. 1941-1964.

[35] D. Li AND J. Rodrigo, Finite-time singularities of an aggregation equation in $\mathbb{R}^{n}$ with fractional dissipation, Comm. Math. Phys., 287(2), (2009), pp. 687-703.

[36] D. Li And J. RodRIgo, Refined blowup criteria and nonsymmetric blowup of an aggregation equation, Advances in Mathematics, 220(1), (2009), pp. 1717-1738.

[37] D. Li AND X. ZhANG, On a nonlocal aggregation model with nonlinear diffusion, (2008) preprint.

[38] H. Li and G. Toscani, Long-time asymptotics of kinetic models of granular flows, Arch. Ration. Mech. Anal. 172, (2004), pp. 407-428.

[39] G. Loeper, Uniqueness of the solution to the Vlasov-Poisson system with bounded density, J. Math. Pures Appl. 86 (2006), pp. 68-79.

[40] A. Majda and A.L. Bertozzi, Vorticity and Incompressible Flow, Cambridge Texts in Applied Mathematics, Cambridge University Press, United Kingdom, 2002. 
[41] R.J. MCCAnN, A convexity principle for interacting gases, Adv. Math. 128, 1 (1997), pp. 153-179.

[42] A. Mogilner And L. Edelstein-Keshet, A non-local model for a swarm, J. Math. Bio. $38,(1999)$, pp. 534-570.

[43] D. Morale, V. Capasso and K. OelschläGer, An interacting particle system modelling aggregation behavior: from individuals to populations, J. Math. Biol., 50 (2005), pp. 49-66.

[44] A. Okubo, S. Levin, Diffusion and Ecological Problems: Modern Perspectives, Springer, Berlin, 2002.

[45] F. Отто, The geometry of dissipative evolution equations: the porous medium equation, Comm. Partial Differential Equations, 26 (2001) pp. 101-174.

[46] C.S. Patlak, Random walk with persistence and external bias, Bull. Math. Biophys., 15 (1953), pp. 311-338.

[47] C.M. Topaz And A.L. BerTOzzI, Swarming patterns in a two-dimensional kinematic model for biological groups, SIAM J. Appl. Math., 65 (2004), pp. 152-174.

[48] C.M. Topaz, A.L. Bertozzi, And M.A. Lewis, A nonlocal continuum model for biological aggregation, Bulletin of Mathematical Biology, 68(7), pp. 1601-1623, 2006.

[49] G. Toscani, One-dimensional kinetic models of granular flows, RAIRO Modél. Math. Anal. Numér., 34, 6 (2000), pp. 1277-1291.

[50] C. Villani, Topics in optimal transportation, Graduate Studies in Mathematics Vol. 58, Amer. Math. Soc, Providence, 2003.

[51] C. Villani, Optimal transport, old and new, Springer, 2008.

[52] V.I. Yudovich, Non-stationary flow of an incompressible liquid, Zh. Vychisl. Mat. Mat. Fiz. 3, (1963), pp. 1032-1066. 